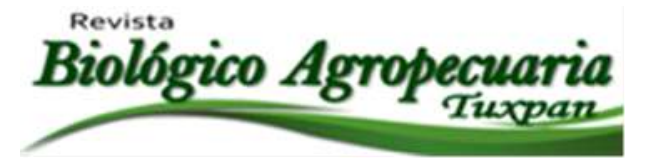

\title{
Fertilidad y producción de caña de azúcar en Hueyapan de Ocampo, Veracruz, México
}

Fertility and production of sugar cane in Hueyapan of Ocampo, Veracruz, Mexico

\author{
Retureta Aponte Alejandro ${ }^{1}$, Hernández Acosta Elizabeth ${ }^{2 \bowtie}$, Salazar Gómez Ana Luisa ${ }^{1}$, Tinoco Alfaro Carlos \\ Alberto $^{1}$, Vázquez Luna Dinora ${ }^{1}$ y Carmona Díaz Gustavo ${ }^{1}$ \\ ${ }^{1}$ Universidad Veracruzana. Facultad de Ingeniería en Sistemas de Producción Agropecuaria, ${ }^{2}$ Universidad \\ Autónoma de Chapingo. Departamento de suelos \\ $\bowtie$ Autor para correspondencia: ehernandeza@chapingo.mx
}

Recibido: $18 / 04 / 2020$

Aceptado: 24/05/2020

\section{RESUMEN}

Se analizaron las principales características químicas para determinar el estado de fertilidad de los suelos tipo Andosol, Vertisol y Gleysol, donde se establece el sistema productivo caña de azúcar en Zapoapan de Amapan perteneciente al municipio de Hueyapan de Ocampo del estado de Veracruz. La importancia de este trabajo radica en ubicar la situación que origina rendimientos muy por debajo de lo que se específica para México. El objetivo del presente trabajo fue evaluar los parámetros $\mathrm{pH}$, materia orgánica, conductividad eléctrica, nitrógeno inorgánico, fósforo, contenidos de potasio, calcio, magnesio, fierro, cobre, manganeso, boro y densidad aparente, siguiendo los métodos establecidos en la NOM-021RECNAT-2000, para ubicar el estado de fertilidad de los suelos y los posibles impactos ambientales ejercidos sobre este recurso natural. Los resultados mostraron que los suelos andosoles tuvieron las mejores condiciones de fertilidad para el cultivo de caña de azúcar; en tanto que los suelos tipo vertisol mostraron problemas leves de contaminación por los contenidos de boro obtenidos. La materia orgánica y el nitrógeno inorgánico son las variables que se deben atender para mejorar la fertilidad de los suelos. La producción de caña de azúcar en la comunidad de Zapoapan de Amapan, es baja (de 25 a 50 ton hectárea), por lo que se recomienda mejorar las prácticas agrícolas con el fin de incrementar los contenidos de nutrimentos en los tres tipos de suelo.

Palabras clave: Suelos, Rendimiento, Sistema productivo.

\begin{abstract}
ABSTRAC
The main chemical characteristics had been analyzed to determine the soil fertility status in the types Andosol, Vertisol and Gleysol where the productive system in sugar cane is established in Zapoapan de Amapan belonging to the town of Hueyapan de Ocampo in the state of Veracruz. The importance of this work lies in placing the situation that origins yields lower that it was expected in Mexico. The goal of the present work was to evaluate the parameters of $\mathrm{pH}$, organic material, electric conductivity, inorganic nitrogen, phosphorous, potassium content, calcium, magnesium, iron, copper, manganese, boron and bulk density, following the established methods in the NOM-021-RECNAT-2000 to place the soil fertility status and the possible environmental impacts exerted on this natural resource. The results showed that Andosol soils had better conditions of fertility for the sugar cane cultivation, however
\end{abstract}


Vertisol soils showed slight contamination problems for the boron contents. The organic material and the inorganic nitrogen are the factors that must attend to enhance the soil fertility. The sugar cane production in the community of Zapoapan de Amapan its lower (from 25 to 50 tons per hectare), so it is recommended to enhance agricultural practices with a view to increment nutritional contents in these three types of soil.

Keywords: Soils, Efficiency, Productive System.

\section{INTRODUCCIÓN}

La disminución de la fertilidad del suelo es el decremento neto de nutrimentos y materia orgánica disponibles, debido a un balance negativo en las entradas y las salidas de ambos elementos y repercute en una baja productividad de los cultivos (SEMARNAT-UACh, 2003).

El manejo de nutrientes debe encaminarse no solamente a lograr rendimientos altos sino también a mantener y elevar la fertilidad del suelo (Tello-Céspedes y Peña-Fonseca, 2019); y aún más en cultivos como la caña de azúcar que requiere cantidades altas de nutrientes en el suelo y dosis de fertilización que incluyan macro y micronutrientes para suplir sus necesidades, debido a su elevada producción de biomasa (Velasco, 2014).

Por lo tanto, la evaluación de la fertilidad es una medida que retoma importancia al brindarnos información que apoye a determinar lo óptimo, o no, que pueden ser los suelos donde establecerán cultivos de interés económico. Las propiedades del suelo que se valoran para ubicar su estado de fertilidad son: contenido de materia orgánica, $\mathrm{pH}$, conductividad eléctrica, macro y micronutrientes, capacidad de intercambio catiónico, densidad aparente y textura, entre otras. En México para evaluar la fertilidad de los suelos, los resultados obtenidos de los análisis en laboratorio se comparan con la NOM021-RECNAT-2000, con esta herramienta se comprueba si se cumple con los límites permisibles para cada uno de los parámetros de interés. Si los valores rebasan y se encuentran por debajo de lo que establece la norma, se debe corregir cada uno de los parámetros que lo requieran, para lograr alta producción de biomasa en los cultivos (Sánchez et al., 2011).

Los suelos de México presentan algún tipo de degradación física, química y biológica, esto se originó debido al mal manejo que se les da y sobre todo por realizar cambios de uso en ecosistemas con una gran biodiversidad. Los cambios de usos más frecuentes se ubican en los bosques y selvas, donde las actividades agropecuarias generan impactos ambientales negativos, entre ellos la pérdida de suelo y biodiversidad (Hernández et al., 2019). Lo anterior origina que se requieran de manejos integrados para mantener y superar el potencial productivo de los suelos sin sacrificar la sustentabilidad de sistema productivo y favorecer la tan requerida seguridad alimentaria (Burbano, 2016).

Uno de los cambios de uso de suelo que se realizan en México es originado por el establecimiento de plantaciones con caña de azúcar cuya producción se realiza de forma extensiva, porque tiene la finalidad de alcanzar altos rendimientos, ejerciendo diversas presiones ambientales en el recurso suelo. El uso inadecuado de los fertilizantes es una tarea que provoca impactos negativos en dicho recurso, esto ocurre porque se aplican dosis inadecuadas debido a que el productor no se apoya de análisis de suelos para agregar la cantidad exacta de nutrimentos que requiere el cultivo de la caña de azúcar. El resultado final es que se pierda la capacidad productiva de los suelos, por lo que requieren aumentar las superficies 
utilizadas para este cultivo, provocando más impactos negativos, y así, continúan produciendo bajo un círculo vicioso.

El esquema anterior se repite en el estado de Veracruz, específicamente en el sur, en donde las instituciones en ciencias agropecuarias pretenden influir positivamente proponiendo un enfoque productivo y sustentable para el cultivo de la caña de azúcar. Por esta razón, el presente estudio se realizó con el objetivo de evaluar y comparar la fertilidad de los suelos donde se cultiva dicha especie en la comunidad de Zapoapan de Amapan municipio de Hueyapan de Ocampo, Veracruz, México, para ubicar su estado actual de fertilidad y los posibles impactos ambientales negativos que se originan con la práctica de fertilización.

\section{MATERIALES Y MÉTODOS}

Descripción del área de estudio. El área de estudio se encuentra ubicada en la comunidad de Zapoapan de Amapan, misma que pertenece a la zona cañera número 5 ubicada en el municipio de Hueyapan de Ocampo, Veracruz, México. El municipio se encuentra entre los paralelos $18^{\circ} 04^{\prime}$ y $18^{\circ} 20^{\prime}$ de latitud norte; los meridianos $94^{\circ} 57^{\prime}$ y $95^{\circ} 25^{\prime}$ de longitud oeste; altitud entre 10 y $800 \mathrm{~m}$. Colinda al norte con los municipios de San Andrés Tuxtla, Catemaco y Soteapan; al este con los municipios de Soteapan y Acayucan; al sur con los municipios de Acayucan y Juan Rodríguez Clara; al oeste con los municipios de Juan Rodríguez Clara, Isla y San Andrés Tuxtla. Ocupa 0.99\% de la superficie del estado y cuenta con 152 localidades y una población total de 38175 habitantes (Figura 1).

Su clima es cálido subhúmedo con lluvias en verano, con temperaturas de 22 a $28^{\circ} \mathrm{C}$ y una precipitación de 1100 a $4100 \mathrm{~mm}$. Los suelos dominantes son: Andosol (44\%), Vertisol (45\%), Gleysol (8\%) y Cambisol (1\%). El uso de suelo y vegetación es: Agricultura (51\%), Zona urbana (1\%), Pastizal (35\%), selva $(11 \%)$ y bosque (1\%). El uso potencial de la tierra es agrícola y pecuario (INEGI, 2009).

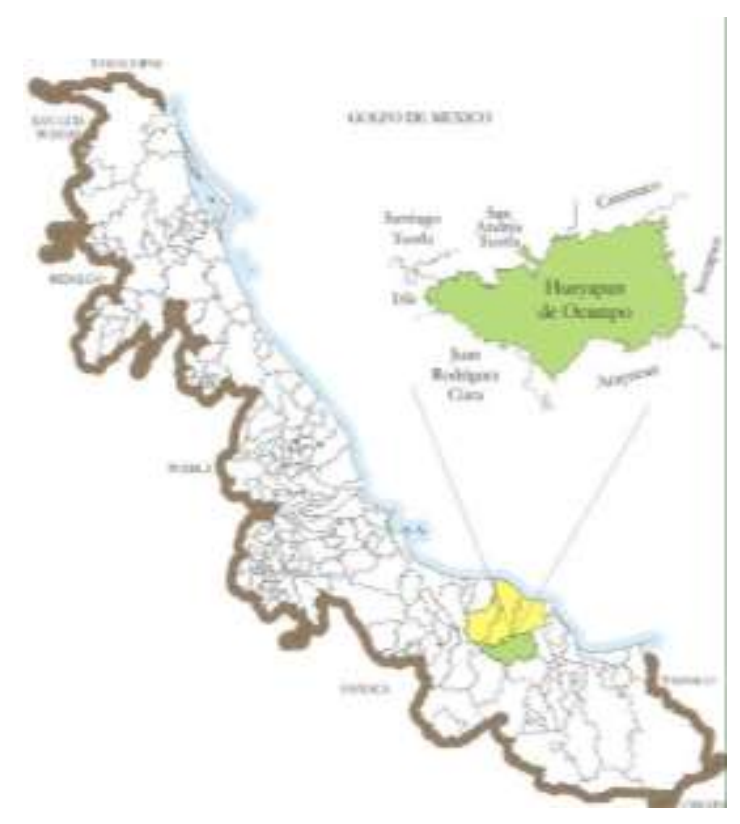

Figura 1. Localización del área de estudio. 
Muestreo de suelo. Los suelos dominantes de la zona cañera del área de estudio se subdividieron en tres tipos: andosoles, vertisoles y gleysoles, en una superficie cultivada aproximada de 443 hectáreas. Para el muestreo se trazó un polígono con la ubicación y división de las diferentes parcelas con cultivo de caña de azúcar para estos tres tipos de suelos, con ayuda de la aplicación Google Earth Pro. En cada tipo de suelo se obtuvieron submuestras siguiendo las recomendaciones de la NOM-021-RECNAT2000 mediante el método zigzag, a una profundidad de $0.30 \mathrm{~cm}$; en total se colectaron 40 submuestras en cada uno de los tres tipos de suelo. Después de aplicar el método de cuarteo se tuvieron tres muestras compuestas con un peso aproximado de $1.5 \mathrm{~kg}$ cada una; las cuales se enviaron para su análisis posterior al laboratorio central del Departamento de Suelos de la Universidad Autónoma de Chapingo, ubicado en Texcoco, Estado de México.

Análisis de Suelos. Con el objetivo de ubicar el estado de fertilidad de los tres tipos de suelo, se evaluaron las propiedades físicas y químicas: $\mathrm{pH}$ (medido con potenciómetro), materia orgánica (procedimiento de Walkley y Black), conductividad eléctrica (extracto de saturación, con potenciómetro), nitrógeno inorgánico (Método micro-Kjeldahl), fósforo (Procedimiento Bray y Kurtz), bases intercambiables $(\mathrm{K}, \mathrm{Ca}$ y $\mathrm{Mg}$ ) (acetato de amonio $1 \mathrm{~N}, \mathrm{pH} 7$ ), micronutrientes ( $\mathrm{Fe}, \mathrm{Cu}, \mathrm{Mn}$ y B) (con EDTA) y, densidad aparente (por el método de parafina). Todos los procedimientos y métodos son los que establece la NOM-021RECNAT-2000.

Manejo del cultivo de caña de azúcar en Juan Díaz Covarrubias, municipio de Hueyapan de Ocampo, Veracruz. Encuestas a productores. Con el objetivo de obtener información sobre las labores que realizan al cultivo de la caña de azúcar se entrevistaron un total de 125 productores, los cuales representan 50\% del total (250 productores), que son los dueños de las 443 hectáreas. Las preguntas que se realizaron fueron: ¿Cuántas toneladas por hectárea produce en promedio en sus cañales?, ¿Su superficie cuenta con riego o es de temporal?, ¿Fertilizan sus suelos?, ¿Qué fertilizantes agregan?, ¿Qué dosis de fertilizante aplica?, y, por último, ¿El ingenio azucarero les sugiere la dosis de fertilizante a aplicar?

\section{RESULTADOS Y DISCUSIÓN}

El estado de fertilidad que tiene cada tipo de suelo donde se cultiva caña de azúcar se describe a continuación.

Suelos Andosoles. El pH que presenta este tipo de suelo es neutro; al respecto Osorio (2012), cita que la caña de azúcar es un cultivo que crece bien en suelos cercanos a la neutralidad. También menciona que este valor permite que los macronutrientes se encuentren disponibles para los cultivos; por lo que no serán una limitante para el desarrollo de la planta y por tanto para su producción. Respecto a la conductividad eléctrica, se observó que este suelo no presenta problemas por concentración de sales ya que tuvo un valor muy por debajo $\left(0.06 \mathrm{dS} \mathrm{m}^{-1}\right)$ del establecido por la norma que es $1 \mathrm{dS} \mathrm{m}^{-1}$.

En cuanto al contenido de materia orgánica, el suelo andosol presentó valores bajos (4.2\%), al respecto, varios estudios han mostrado que los suelos donde se cultiva caña de azúcar tienen bajos contenidos de materia orgánica, generalmente de 3 a $4 \%$, lo cual se convierte en un problema a corregir para que su producción no sea baja (Cabrera y Zuaznábar, 2010).

Los contenidos de nitrógeno (7.0 $\mathrm{mg} \mathrm{kg}^{-1}$ ) y potasio (1.07 mg kg-1) fueron bajos, y aunque el 
$\mathrm{pH}$ del suelo no es una limitante para su absorción por las raíces, se requerirá incorporarlo para que la producción del cultivo no sea baja. De los dos elementos el nitrógeno suele ser el más deficiente. Lo anterior lo fundamenta López-Hernández et al. (2005), quienes estudiaron los balances de nitrógeno en un agroecosistema de caña de azúcar y concluyeron que la cantidad de nitrógeno presente en el suelo disminuyó a razón de $5.3 \mathrm{~kg}$ $\mathrm{N}$ ha ${ }^{-1}$ año ${ }^{-1}$ con la lixiviación, así como por las cosechas y por quemas, explico que anualmente 98\% del nitrógeno acumulado en la biomasa aérea, se pierde por estas dos vías. La corrección de este elemento en el suelo en México se realiza mediante la aplicación de urea.

El contenido de fósforo fue medio $\left(24.06 \mathrm{mg} \mathrm{kg}^{-}\right.$ $\left.{ }^{1}\right)$ y el de calcio $\left(16.33 \mathrm{mg} \mathrm{kg}^{-1}\right.$ ) y magnesio (12.28 $\left.\mathrm{mg} \mathrm{kg}^{-1}\right)$ alto. Los contenidos de fierro, cobre, zinc y manganeso fueron adecuados y el boro en el suelo tuvo un contenido bajo (Cuadro 1). La densidad aparente obtenida para este tipo de suelo corresponde a una textura Franca, ideal para el crecimiento del cultivo, porque favorece la movilidad de nutrimentos en la solución del suelo (Gisbert-Blanquer et al., 2010)

Cuadro 1. Valores de fertilidad de macro y micronutrientes y su relación con la NOM

\begin{tabular}{|c|c|c|c|c|c|c|c|}
\hline Tipo de Suelo & $\mathrm{pH}$ & $\begin{array}{l}\text { C.E. } \\
\mathrm{dS} \mathrm{m} \mathrm{m}^{-1}\end{array}$ & $\underset{\%}{\text { M.O. }}$ & $\begin{array}{c}\mathrm{N} \\
\text { inorgánico } \\
\mathrm{mg} \mathrm{kg}^{-1}\end{array}$ & $\begin{array}{c}\mathrm{P} \\
\mathrm{mg} \mathrm{kg}^{-1}\end{array}$ & $\begin{array}{c}\mathrm{K} \\
\mathrm{Cmol}(+) \\
\mathrm{kg}^{-1}\end{array}$ & $\begin{array}{c}\mathrm{Ca} \\
\mathrm{Cmol} \mathrm{(+)} \\
\mathrm{kg}^{-1}\end{array}$ \\
\hline ANDOSOLES & 6.61 & 0.06 & 4.28 & 7.0 & 24.06 & 1.07 & 16.33 \\
\hline VERTISOLES & 7.10 & 0.06 & 3.92 & 7.0 & 23.20 & 0.50 & 14.14 \\
\hline GLEYSOLES & 6.31 & 0.06 & 4.28 & 8.8 & 17.00 & 0.41 & 16.33 \\
\hline \multirow[t]{2}{*}{$\begin{array}{c}\text { NOM-021- } \\
\text { RECNAT-2000 }\end{array}$} & $\begin{array}{l}\text { Neutro } \\
(6.6-7.3)\end{array}$ & $\begin{array}{c}\text { Efectos } \\
\text { despreciables } \\
\text { de salinidad } \\
\text { (menores 1.0) }\end{array}$ & $\begin{array}{c}\text { Bajo } \\
(4.1-6.0)\end{array}$ & $\begin{array}{l}\text { Muy bajo } \\
(0-10)\end{array}$ & $\begin{array}{l}\text { Medio } \\
(15-30)\end{array}$ & $\begin{array}{l}\text { Alta } \\
\text { (mayor } \\
10)\end{array}$ & $\begin{array}{c}\text { Alta } \\
\text { (mayor 10) }\end{array}$ \\
\hline & $\begin{array}{c}\mathrm{Mg} \\
\mathrm{cmol}^{(+)} \\
\mathrm{Kg}^{-1}\end{array}$ & $\mathrm{Fe}$ & $\mathrm{Cu}$ & $\begin{array}{c}\mathrm{Zn} \\
\mathrm{mg} \mathrm{kg}^{-1}\end{array}$ & Mn & B & $\begin{array}{c}\text { Densidad } \\
\text { Aparente } \\
\mathrm{gr} / \mathrm{cm}^{3}\end{array}$ \\
\hline ANDOSOLES & 12.28 & 100.82 & 8.98 & 3.32 & 55.38 & 0.76 & 1.30 \\
\hline VERTISOLES & 10.90 & 72.27 & 8.63 & 2.42 & 50.67 & 1.00 & 1.19 \\
\hline GLEYSOLES & 11.83 & 171.90 & 17.28 & 1.81 & 53.10 & 0.68 & 1.28 \\
\hline $\begin{array}{c}\text { NOM-021- } \\
\text { RECNAT-2000 }\end{array}$ & $\begin{array}{l}\text { Alta } \\
\text { (mayor } \\
3.0 \text { ) }\end{array}$ & $\begin{array}{l}\text { Adecuado } \\
\text { (mayor 4.5) }\end{array}$ & $\begin{array}{l}\text { Adecuado } \\
\text { (mayor } \\
0.2 \text { ) }\end{array}$ & $\begin{array}{l}\text { Adecuado } \\
\text { (mayor 1.0) }\end{array}$ & $\begin{array}{c}\text { Adecuado } \\
\text { (mayor } \\
1.0 \text { ) }\end{array}$ & $\begin{array}{l}\text { Bajo } \\
(0.39- \\
0.79)\end{array}$ & $\begin{array}{c}\text { Franco } \\
(1.20-1.32)\end{array}$ \\
\hline
\end{tabular}


Suelos Vertisoles. Al igual que para el suelo andosol, este suelo no presentó problemas de acidez, ni alcalinidad, el pH que se obtuvo fue neutro, por lo que la disponibilidad de nutrimentos para el cultivo de caña de azúcar no será una limitante. La conductividad eléctrica tampoco representó un problema, ya que se encontró un contenido despreciable de salinidad (Cuadro 1).

El contenido de materia orgánica (3.92\%), nitrógeno inorgánico $\left(7.0 \mathrm{mg} \mathrm{kg} \mathrm{kg}^{-1}\right)$ y potasio (0.50 mg kg-1) fueron bajos, por lo que, para lograr una buena producción del cultivo será importante corregir los problemas de deficiencias de estas variables durante el ciclo de vida del cultivo (Cabrera y Zuaznábar, 2010). Este comportamiento no siempre es común en los suelos vertisoles donde se cultiva la caña de azúcar, al respecto, Ribón et al. (2003) encontraron resultados diferentes a los obtenidos en el presente estudio; los investigadores evaluaron en suelo vertisol cultivado con caña de azúcar durante 30 años y encontraron contenidos ricos en materia orgánica $(3.65 \%)$ y nitrógeno $(0,19 \%)$ y contenido medio para potasio $(0,3$ a $\left.0,6 \mathrm{cmol} \cdot \mathrm{kg}^{-1}\right)$.

Para los elementos fósforo, calcio y magnesio, los resultados indicaron que se encontraron contenidos medios ( $\mathrm{P}, 23.20 \mathrm{mg} \mathrm{kg} \mathrm{m}^{-1}$ ) y altos (Ca, $14.14 \mathrm{mg} \mathrm{kg}^{-1}$ y Mg $10.90 \mathrm{mg} \mathrm{kg}^{-1}$ ). Al igual que en los suelos andosoles, los contenidos de fierro, cobre, zinc y manganeso fueron adecuados, no representan problemas para el desarrollo de las plantas porque son requeridos en cantidades pequeñas; tampoco generan problemas de contaminación en el suelo. Situación que difiere con los resultados obtenidos para boro, ya que con este se rebasa el límite establecido por la NOM-021 que es de $0.79 \mathrm{mg} \mathrm{kg}^{-1}$.
Respecto al boro, se ubica que este elemento se requiere en pequeñas cantidades en la producción de caña de azúcar, sin embargo, puede ser tóxico para las plantas, al afectar algunos procesos fisiológicos como la fotosíntesis y el metabolismo de pigmentos (Moreno-Echeverry et al., 2016). Se ha detectado que el boro con facilidad pasa de ser un elemento deficiente a un nutriente tóxico, sobre todo si las prácticas del riego, aplicación de fertilizantes y de lodos residuales se hacen sin considerar su comportamiento en el suelo (Martínez-Orozco et al., 2018).

El suelo vertisol presentó una densidad aparente propia de un suelo Franco, ocupan $8.3 \%$ del territorio nacional y se les consideran los más productivos de México, sin embargo, se ubica que en la zona costera del Golfo de México donde son dominantes, se presentan serios problemas de degradación (Torres-Guerrero et al., 2016). El presente trabajo se ubica en esta zona, por lo que estudios como este son necesarios para reconocer cual es el estado actual del suelo, respecto a sus propiedades físicas y químicas.

Suelos Gleysoles. Para este tipo de suelo a diferencia de los dos anteriores se presenta con un $\mathrm{pH}$ débilmente ácido; esto es normal de acuerdo con lo que señala Bernal et al. (2015), estos valores predominan en los suelos de las partes más bajas porque a medida que aumenta la gleyzación el pH es ácido. Respecto a la Conductividad eléctrica, se observó que este suelo tampoco tiene problemas por concentración de sales ya que tuvo un valor muy por debajo $\left(0.06 \mathrm{dS} \mathrm{m}^{-1}\right)$ del establecido por la norma que es $1 \mathrm{dS} \mathrm{m}^{-1}$ (Cuadro 1 ).

Respecto a la materia orgánica, este tipo de suelos presentó valores bajos $(4.2 \%)$. Siendo normal estos valores porque como lo señala 
Ibañez (2011) que estos suelos atesoran un alto contenido de materia orgánica para su producción en los cultivos. En lo que se refiere al contenido de nitrógeno $\left(8.8 \mathrm{mg} \mathrm{kg}^{-1}\right)$ y potasio $\left(0.41 \mathrm{mg} \mathrm{kg}^{-1}\right)$. éstos fueron bajos, seguramente se debe al efecto del $\mathrm{pH}$, el cual genera acidez y esto a su vez se convierte en factor limitante para la disponibilidad de ambos nutrientes. Así mismo el contenido del P fue medio (24.06 mg $\mathrm{kg}^{-1}$ ). Estos nutrientes primarios podrían ser elevados si los productores manejan enmiendas agrícolas, como las que uso Hernández et al., (2008) en su estudio para suelos de tipo Gleysoles en caña de azúcar, quienes a través de la aplicación de vinaza y composta de cachaza no afectaron el $\mathrm{pH}$ y el contenido de materia orgánica, potasio y fósforo se incrementó, por lo tanto, resultó una alternativa viable para el aprovechamiento de ambos subproductos, sin alterar el ambiente y con posibilidad de que su uso pueda satisfacer la demanda nutricional del cultivo de la caña de azúcar.

El contenido de calcio (16.33 $\mathrm{mg} \mathrm{kg}^{-1}$ ) y magnesio (11.83 $\mathrm{mg} \mathrm{kg} \mathrm{kg}^{-1}$ ) es alto. Los contenidos de fierro, cobre, zinc y manganeso fueron adecuados y el boro en el suelo tuvo un contenido bajo (Cuadro 1). La densidad aparente obtenida para este tipo de suelo corresponde a una textura Franca, ideal para el crecimiento del cultivo, porque favorece la movilidad de nutrimentos en la solución del suelo (GisbertBlanquer et al., 2010).

\section{Manejo del cultivo, resultado de las encuestas.}

Como resultado de las encuestas se supo que la producción del cultivo de caña de azúcar en la localidad de Zapoapan de Amapan, en el municipio de Hueyapan de Ocampo, Veracruz varia de 25 a 30 toneladas por hectárea. Se ubicó que en general los productores no fertilizan sus suelos y cuando realizan esta práctica, logran aumentar la producción hasta 50 toneladas por hectárea.

Estos rendimientos son muy bajos, de acuerdo con lo que establecen los Fideicomisos Instituidos en Relación con la Agricultura (FIRA), que en promedio indica deben ser 124 toneladas por hectárea; y recomiendan que, para el cultivo de la caña de azúcar, se debe aplicar por hectárea las siguientes dosis nutrimentales específicas: $235 \mathrm{~kg}$ nitrógeno, $112 \mathrm{~kg}$ fósforo, $370 \mathrm{~kg}$ potasio, $31 \mathrm{~kg}$ magnesio y $28 \mathrm{~kg}$ azufre. Lo anterior coincide con lo señalado por Quintero (1995), quien indica que se requieren grandes cantidades de nutrientes para el crecimiento y producción de este cultivo y que se deben ejecutar métodos de aplicación de fertilizantes que mejoren la eficiencia de absorción de dichos nutrimentos por las plantas.

\section{CONCLUSIONES}

Se ubicó que los suelos andosoles presentaron las mejores condiciones de fertilidad para el cultivo de la caña de azúcar, seguidos de los gleysoles y vertisoles, en este último se supo que existen problemas leves en el contenido de boro, por lo que se sugiere informar a los productores para que tomen los cuidados correspondientes.

De las propiedades químicas del suelo, se supo que la materia orgánica y el nitrógeno inorgánico son las variables que se deben atender para mejorar la fertilidad del mismo, ya sea mediante una fertilización inorgánica adecuada o con la incorporación de enmiendas orgánicas como la cachaza y el estiércol.

La producción de caña de azúcar en la comunidad de Zapoapan de Amapan, municipio de Hueyapan de Ocampo es baja, con aplicación de fertilizantes inorgánicos $(50$ toneladas hectárea) y sin fertilización (25 toneladas por hectárea). 
Por la situación anterior, se recomienda mejorar las prácticas agrícolas en el sistema de producción del cultivo de caña de azúcar, de Zapoapan de Amapan, municipio de Hueyapan de Ocampo, enfáticamente en la fertilización.

\section{LITERATURA CITADA}

Bernal, A; Hernández, A.; Mesa, M; Rodríguez, O; González, P. J; Reyes, R. 2015. Características de los suelos y sus factores limitantes de la región de murgas, provincia la habana. Cultivos Tropicales. Instituto Nacional de Ciencias Agrícolas La Habana, Cuba. 36(2). 30-40.

Burbano, O. H. 2016. El suelo y su relación con los servicios ecosistémicos y la seguridad alimentaria. Revista de Ciencias Agrícolas, 33(2), $117-$ 124. https://dx.doi.org/10.22267/rcia.16 3302.58

Cabrera, J. A; \& Zuaznábar, R. 2010. Impacto sobre el ambiente del monocultivo de la caña de azúcar con el uso de la quema para la cosecha y la fertilización nitrogenada. I. Balance del Carbono. Cultivos Tropicales, 31(1), 513. Recuperado en 16 de abril de 2020, de

http://scielo.sld.cu/scielo.php?script=sci _arttext\&pid=S02585936201000010000 $1 \& \operatorname{lng}=$ es \&tlng=es.

Cabrera, J. A; \& Zuaznábar, R. 2010. Respuesta de la caña de azúcar a la fertilización nitrogenada en un experimento de Larga Duración con 24 cosechas acumuladas. Cultivos Tropicales, 31(1), 93-100. Recuperado en 17 de abril de 2020, http://scielo.sld.cu/scielo.php?script=sci _arttext\&pid=S0258$59362010000100014 \& \operatorname{lng}=\mathrm{es} \& \operatorname{tlng}=\mathrm{pt}$

FAO/UNESCO/ISRIC. 1998. Organización para la alimentación y la agricultura de naciones unidas. Sistema de clasificación de suelos. Base referencial mundial.

FIRA. 2010. Fideicomisos Instituidos en Relación con la Agricultura. Producción Sostenible de Caña de Azúcar en México en Boletín Informativo, Nueva Época, Número 11.

Gisbert B; J. M; Ibáñes A. S; \& Moreno R. H. 2010. La Textura de un Suelo. Valencia, España: Escuela Técnica Superior de Ingenieros Agrónomos, Universidad Politécnica de Valencia. 1-8.

Hernández, M. G. I; Salgado G. Sergio; Palma L. D. J; Lagunes L. del C; C, E. M; Ruiz, R. Octavio. 2008. Vinaza y composta de cachaza como fuente de nutrientes en caña de azúcar en un gleysol mólico de Chiapas, México Interciencia. Asociación Interciencia Caracas, Venezuela 33(11), 860-855.

Hernández A. E. y Segura. L. J. 2018. Suelos de bosque mesófilo de montaña degradados por actividades de cambio de uso. Memorias del $43^{\circ}$ Congreso Nacional de la Ciencia del Suelo, Buenavista, Saltillo, Coahuila, México.

Ibañez, J. J. y Manriquez, C. F. J. 2011. Gleysoles: uso y manejo. Un universo invisible bajo nuestros pies: los suelos y la vida. Fundación para el conocimiento. Madrid España. De http://www.madrimasd.org/blogs/universo /2011/02/25/138174 
INEGI. 2009. Instituto Nacional de Estadística y Geografía e Información. Carta de suelos dominantes del Prontuario de información geográfica municipal de los Estados Unidos Mexicanos.

López, H., D; Infante, C. y Medina, E. 2005. Balance de elementos en un agroecosistema de caña de azúcar: I. Balance de nitrógeno. Tropicultura, 23, 212-219.

Martínez, O. E; González A. I. J; De La Mora O. C; Flores, L. H. E; Chávez, D. A. A. 2018. Nutrimentos en caña de azúcar (Saccharum officinarum) bajo condiciones de estrés por salinidad. Memorias del $43^{\circ}$ Congreso Nacional de la Ciencia del Suelo Buenavista, Saltillo, Coahuila, México.

Moreno, E. D; Quiroga, I. A; Balaguera, L. H. E; y M. Stanislav. 2016. El estrés por boro afecta la fotosíntesis y el metabolismo de pigmentos en plantas: Revista Colombiana De Ciencias Hortícolas. $10 \quad$ (1): 137148. https://doi.org/10.17584/

rcch.2016v10i1.4189

NOM-021-SEMARNAT-2000. Secretaría de Medio Ambiente y Recursos Naturales. Norma Oficial Mexicana 021. Especificaciones de fertilidad, salinidad, y clasificación de suelos, estudio, muestreo y análisis.

Osorio, N. W. 2012. pH del suelo y disponibilidad de nutrientes. Manejo Integral del Suelo y Nutrición Vegetal, 1(4), 1-4. Recuperado de https://www.bioedafologia. $\mathrm{com} /$ sites/default/fles/documentos/pdf/p H-del-suelo-y-nutrientes.pdf

Quintero, D. R. 1995. Fertilización y nutrición: El cultivo de la caña en la zona azucarera de Colombia. CENICAÑA. 153-177.

Ribón, M. A; \& Salgado, G. S; \& Palma, L. D. J; \& Lagunes, E. L. C. 2003. Propiedades químicas y físicas de un vertisol cultivado con caña de azúcar. Interciencia, $\quad$ 28(3),154-159. Disponible en: https://www.redalyc.org/articulo.oa? id $=339 / 33907805$

Sánchez, S. H. M; \& Ruz, F. 2011. Alternativas de manejo de la fertilidad del suelo en ecosistemas agropecuarios. Pastos y Forrajes, 34(4), 375-392. http://scielo.sld.cu/scielo.php?script=sci _arttext\&pid=S08640394201100040000 $1 \& \operatorname{lng}=\mathrm{es} \& \mathrm{t} \operatorname{lng}=\mathrm{pt}$.

SEMARNAT y UACh. 2003. Secretaria de Medio Ambiente y Recurso Naturales y la Universidad Autónoma de Chapingo. El medio ambiente en México. Evaluación de la pérdida de suelos por erosión hídrica y eólica en la República Mexicana, escala 1: 1000 000. Memoria 2001-2002. México.

Tello, C. R; \& Peña, F. L. 2019. Servicio de recomendaciones de fertilizantes y enmiendas, una alternativa para la aplicación racional de los fertilizantes minerales en caña de azúcar. InfoCiencia, 20(1), 9. Recuperado de http://www.infocienciass.cu/index.php/in fociencia/article/view/153 
Torres, G. C. A; Gutiérrez, C. M. C; Ortiz, S. C. A; \& Gutiérrez, C. E. V. 2016. Agricultural management of Vertisols in Mexico: a review. Terra Latinoamericana, 34(4), 457-466.

http://www.scielo.org.mx/scielo.php?scr ipt=sci_arttext\&pid=S01875779201600 0400457\&lng=es\&tlng=en.
Velasco, V. J. 2014. Los biofertilizantes y la producción de caña de azúcar (Saccharum Spp.), México: Colegio de Postgraduados. México. 7 (2). 60-64.

Copyright (c) 2020 Retureta Aponte Alejandro, Hernández Acosta Elizabeth, Salazar G ómez Ana Luisa, Tinoco Alfaro Carlos Alberto, Vázquez Luna Dinora y Carmona Diaz Gustavo

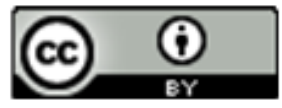

Este texto está protegido por una licencia licencia CreativeCommons 4.0 .

Usted es libre para Compartir —copiar y redistribuir el material en cualquier medio o formato- y Adaptar el documento —remezclar, transformar y crear a partir del material- para cualquier propósito, incluso para fines com erciales, siempre que cumpla la condición de:

Atribución: Usted debe dar crédito a la obra original de manera adecuada, proporcionar un enlace a la licencia, e in dicar si se han realizado cambios. Puede hacerlo en cualquier forma razonable, pero no de forma tal que sugiera que tiene el apoyo del licenciante o lo recibe por el uso que hace de la obra.

Resumendelicencia - Textocompletodelalicencia 